Rapport - Société canadienne d'histoire de l'Église catholique

\title{
Monseigneur Bourget et la Confédération
}

\section{Léon Pouliot}

Volume 26, 1959

URI : https://id.erudit.org/iderudit/1007412ar

DOI : https://doi.org/10.7202/1007412ar

Aller au sommaire du numéro

Éditeur(s)

La Société canadienne d'histoire de l'Église catholique

ISSN

0318-6148 (imprimé)

1927-7075 (numérique)

Découvrir la revue

Citer cet article

Pouliot, L. (1959). Monseigneur Bourget et la Confédération. Rapport - Société canadienne d'histoire de l'Église catholique, 26, 31-41.

https://doi.org/10.7202/1007412ar

Tous droits réservés ( La Société canadienne d'histoire de l'Église catholique, 1960
Ce document est protégé par la loi sur le droit d'auteur. L'utilisation des services d’Érudit (y compris la reproduction) est assujettie à sa politique d'utilisation que vous pouvez consulter en ligne.

https://apropos.erudit.org/fr/usagers/politique-dutilisation/ 


\section{Monseigneur Bourget et la Confédération}

\section{Avant L'ÉvÉNEMENT}

Au printemps de 1864, le parti conservateur, dont Georges-Etienne Cartier était le chef dans le Bas-Canada, faisait de la Confédération un article, le principal article de son programme. Le sujet n'était pas nouveau. En 1857-1858, dans le Courrier du Canada, Joseph-Charles Taché en avait souligné les nombreux avantages ${ }^{1}$. De plus, on espérait sortir par là de l'impasse où l'instabilité des gouvernements plongeait à tout instant les Canadas-Unis. L'envergure du projet, les circonstances dans lesquelles il avait pris corps, c'est-à-dire l'alliance de Cartier avec George Brown, chef clear-grit du Haut-Canada et implacable ennemi des Canadiens français, étaient de nature à passionner les esprits. Et, en effet, tenus en haleine par les journaux partisans, les esprits se passionnent.

Non seulement on veut la Confédération; on la veut tout de suite. De là cette délégation des Canadas-Unis dans les Provinces Maritimes; de là les Conférences de Québec qui ont lieu à l'automne de 1864, et où les délégués des provinces intéressées arrêtent les clauses du projet.

Entre la visite des représentants du Canada aux Provinces Maritimes et les Conférences de Québec, un fait est à noter où l'on voit que le parti conservateur entend mener rondement les choses et façonner l'opinion publique : l'organe des conservateurs, La Minerve, qui paraissait jusque-là trois fois la semaine, devient quotidien. Et il ne cache pas les raisons de cette publication plus fréquente. Relisons ensemble son article du 9 septembre :

De nouveaux changements se préparent pour notre pays; la position qui nous a été faite par l'Union n'est pas soutenable, et il est devenu nécessaire de prévenir une rupture que pourrait faire naître une situation trop tendue. La Confédération a été proposée comme devant remédier aux difficultés graves qui entravent, à chaque session, la législation du pays. Nous avons exprimé notre confiance dans le principe de cette mesure, et nous avons cru qu'elle était destinée à préparer les voies à l'avenir de paix et de gloire que nous désirons tous pour notre pays. Mais, en même temps, nous comprenons toute la gravité de cette mesure, qui doit être probablement la dernière pierre à la consolidation de notre édifice politique. Nous avons confiance dans les hommes aujourd'hui à la tête du pouvoir, et nous croyons cette confiance méritée. Aussi, sans former aucun engagement à l'avance, nous attendons avec espoir le projet qui résultera de leurs délibérations et de leurs études.

$\mathrm{Si}$ nous croyons voir dans ce projet la sauvegarde des intérêts particuliers du Bas-Canada, de sa religion et de sa nationalité, nous lui donnerons notre appui. Sinon, nous le combattrons de toutes nos

1 Articles publiés en forme de volume: . Des Provinces de l'Amérique du Nord et d'une Union fédérale. " Québec, Brousseau, 1858, $254 \mathrm{pp}$. 
forces. Il est une chose que nous mettons toujours au-dessus des questions d'hommes et de partis : c'est la cause de la nationalité.

Il y a trente-sept ans, La Minerve donnait cette assurance, en s'adressant pour la première fois au public. Au milieu des difficultés graves où se trouve aujourd'hui le pays, et en commençant cette nouvelle série de notre publication, nous renouvelons cette promesse. Elle a été fidèlement tenue dans le passé; nous avons la confiance que c'est une garantie pour l'avenir.

En cet automne de 1864, si gros de conséquences pour l'avenir politique du Bas-Canada, $\mathbf{M}^{\mathbf{g r}}$ Bourget est tout entier aux préparatifs de son cinquième voyage en Europe. Les deux problèmes qu'il va soumettre à l'attention du Saint-Siège sont d'une importance capitale pour la ville et le diocèse de Montréal : fondation d'une université catholique, démembrement de la paroisse de Notre-Dame; le premier a pour adversaire l'Université Laval et le second le Séminaire de Montréal. La lutte, $\mathbf{M}^{\mathrm{gr}}$ Bourget le sait, sera longue et pénible. Raison de plus pour bien fourbir ses armes, pour constituer sur chaque problème une documentation complète, claire, solide. Et ce travail s'ajoute à l'administration d'un vaste diocèse, administration déjà lourde, l'évêque de Montréal n'ayant pas de coadjuteur.

Cela ne l'empêche pas de suivre les événements qui se passent sous ses yeux et qui changeront peut-être la face du pays. Mais il ne se hâte pas de prendre parti. Dans la circulaire qu'il adresse au clergé, le jour même de son départ pour Rome, 14 novembre, il se contente d'écrire :

Nous prierons d'une manière particulière pour le pays, dans ce temps de mouvement et de réforme constitutionnelle, afin que la divine Providence, qui a toujours veillé sur ses intérêts avec une bonté maternelle, dirige toutes les opérations de nos hommes publics, et qu'il ne nous arrive rien qui puisse nuire à la Religion et au bienêtre de notre chère patrie 2 .

Il a, sur l'intervention du clergé dans la chose politique, une attitude plutôt sévère. En 1859, M. Bourgeault, curé de Pointe-Claire, avait adressé à L'Ordre, journal bien pensant, une lettre parfaitement orthodoxe, à l'occasion d'un bill de divorce. $\mathbf{M}^{\mathrm{gr}}$ Bourget n'en permet pas la publication. Il appartient à l'évêque de donner au peuple les avis que vous lui donnez, écrit-il. Et il ajoute : « Je saisis cette occasion, mon cher curé, pour vous faire observer que nous n'avons pas mission pour parler dans les gazettes. ${ }^{3}$ » Aussi, lorsqu'en juillet 1864, un curé de la campagne ose adresser une correspondance à La Presse, journal anti-fédéraliste, l'évêque intervient. En son nom, le secrétaire de l'évêché, M. J.-O. Paré, écrit à l'éditeur de La Presse. Aucun jugement n'est porté sur le contenu de la correspondance; mais en prenant part au débat, le Curé de la campagne a manqué à la discipline imposée au clergé. On est prié de ne pas juger celui-ci par celui-là 4 .

Enfin, sur l'attitude de $\mathbf{M}^{\mathrm{gr}}$ Bourget jusqu'à son départ pour Rome [14 novembre 1864], nous avons le témoignage de son grand-

2 Mand. des év. de Montréal, V, 33.

3 AAM, Lettres de Mor Bourget, 10, 479.

4 Lettre reproduite dans La Minerve du 12 juillet 1864. 
vicaire, administrateur du diocèse. Le 20 février 1865, M. Truteau écrit à M. Cazeau, Vicaire-Général de Québec :

M. Cartier, m'a-t-on dit (car je n'ai pas eu le temps de voir cela par moi-même sur les journaux) aurait dit, en pleine Chambre, que les plus hauts dignitaires des Eglises de chaque diocèse du Canada étaient en faveur de la Confédération. Je ne sais ce que les autres évêques pensent là-dessus; mais quant à $\mathrm{M}^{\mathrm{gr}}$ de Montréal, $\mathrm{je}$ suis positif à dire qu'il n'a pas voulu se prononcer jusqu'ici sur cette question. Avant de partir pour Rome, il disait qu'il aimait mieux attendre avant de prendre aucun parti là-dessus 5 .

On ne voit pas qu'il ait profité de son séjour à Rome pour intéresser le Saint-Siège au problème. Et pourtant, Pie IX est au courant. Le 28 mars 1865, l'évêque de Montréal raconte l'audience que lui a accordée le Pape. Il le félicite et le remercie de l'Encyclique Quanta Cura et du Syllabus :

Là-dessus, il me dit avec ce ton de calme et de confiance qui, ici, rassure tout le monde, qu'il prévoit que cette Lettre [Quanta cura] produirait son fruit avec le temps. Et je fus étonné de l'entendre m'ajouter qu'il espérait beaucoup que les catholiques du Canada se déclareraient ouvertement et fortement pour la défense des lois dont on fait la codification et dont ils doivent soutenir le maintien pour la protection de la religion 6. Il est étonnant de l'entendre parler, lui qui est assiégé de tant d'affaires qui lui passent chaque jour par les mains, de ce qui se passe dans notre pays, de la codification, comme je viens de vous le dire, de la Confédération, de la guerre américaine ${ }^{7}$.

Cet éloge de la codification de nos lois adressé par Pie IX à Mgr Bourget n'était pas un effet du hasard. Il était voulu. Mais qui donc inspirait le Pape ? On pense naturellement à $\mathbf{M}^{\mathrm{gr}}$ Horan, évêque de Kingston et à M. Taschereau, Recteur de l'Université Laval, qui sont également à Rome au début de 1865. Il est certain, en tout cas, que ceux-ci ont soumis aux canonistes romains le cas de conscience que posait aux députés catholiques du Bas-Canada le problème de la Confédération ${ }^{8}$. Il paraît certain également que Mgr Bourget a gardé

5 AAM, Lettres de Mor Bourget, 14, 288-290.

6 Notre code civil, car c'est bien de lui qu'il s'agit, n'était pas pur de tout gallicanisme. Les évêques s'en apercevront plus tard. Témoin le $13^{\circ}$ décret du 4 ${ }^{*}$ concile provincial de Québec (1868) : . Cum in codice civili Canadæ inferioris quidam articuli non satis congruere videantur doctrinæ et disciplinæ Ecclesiæ de Matrimonio, statuimus petitionem præstandam esse Gubernio ab Episcopis ejusdem Provinciæ ut corrigantur iidem articuli., Ce décret n'eut pas de suite pour des raisons qu'il serait trop long d'exposer ici.

7 AAM, Voyage à Rome, 1864-1865, cart. 15.

8 Une clause du projet de confédération enlevait aux gouvernements provinciaux pour les confier au gouvernement fédéral toutes les questions relatives au mariage et au divorce. Pour les Provinces Maritimes, où le divorce était déjà reconnu par la loi, il s'agissait d'un simple transfert de juridiction. Pour les Canadas-Unis, où le divorce n'était pas admis, il en allait autrement; et cette clause introduisait dans notre législation un principe intrinsèquement mauvais. Or, les députés étaient appelés à se prononcer sur le projet en bloc et non sur chaque clause séparément. Dès lors, et quelque recommandable que soit par ailleurs la confédération, les catholiques peuvent-ils par leur vote collaborer à introduire le divorce dans notre législation? Tel est le cas de conscience que propose $M$. Truteau, administrateur du diocèse de Montréal. 
à Rome la prudente neutralité qu'il s'est imposée dès le début; il n'a rien fait pour intéresser le Saint-Siège à la Confédération. Et telle sera son attitude jusqu'à la fin, c'est-à-dire jusqu'au jour où par la volonté du Parlement impérial, la Confédération deviendra le gouvernement légitime du pays. On en a la preuve dans l'accusé de réception, adressé le 11 mars 1867, à Georges-Etienne Cartier, alors à Londres :

Je reçois à l'instant la copie du British North America Bill, que vous avez eu la bonté de m'adresser; et je m'empresse de vous présenter mes sincères remerciements pour votre bienveillante attention.

Je comprends vivement que ce bill intéresse à un haut degré notre pays qui, après toutes les phases d'administration par lesquelles il lui a fallu passer depuis un certain nombre d'années, a grand besoin de se fixer sur des bases solides et durables.

Il serait superflu de vous dire que le clergé, tout en se mettant en dehors de toutes les luttes des partis politiques, n'en est pas moins attaché au pays qui l'a vu naître, et qu'il aime, comme un bon enfant sa mère, et cet amour est d'autant plus ardent qu'il lui est inspiré par la religion ${ }^{9}$.

C'est à dessein, croyons-nous, que le mot de Confédération n'a pas été employé. Bien plus, le problème étant d'ordre politique, le clergé n'a pas à intervenir, sans que l'on puisse pour cela suspecter la qualité de son patriotisme.

\section{APRÈs L'ÉvÉNEMENT}

Jusqu'à la sanction par la Reine du projet de Confédération, il était permis de s'y opposer sans encourir la note d'infidélité ou de désobéissance à l'autorité légitime. Après ce geste décisif, la Confédération devient le gouvernement légitime, que tous doivent accepter,

A Québec on voyait les choses d'un autre œil. Le Courrier du Canada, champion de la confédération, soutenait qu'il s'agissait d'un simple transfert de juridiction. Et c'est ainsi que le cas de conscience fut soumis par $\mathbf{M}^{\mathrm{gr}}$ Horan et par M. Taschereau aux canonistes romains. Sur ces données, ceux-ci répondirent que les députés catholiques pouvaient voter en faveur de la confédération, en toute tranquillité de conscience; par là, en effet, ils ne collaboraient pas à l'introduction d'un principe intrinsèquement mauvais; ils en rendaient - on s'en flattait du moins - l'application plus difficile. La réponse des canonistes romains n'exerça aucune influence sur le vote de l'assemblée. Celle-ci avait déjà accepté le projet de confédération quand celle-là fut connue et rendue publique dans les journaux du pays. M. Truteau n'en voulut pas moins réitérer la position de Montréal. Il écrivait à M. Cazeau, le 3 avril 1865 : Ce qui a engagé les théologiens de Rome à donner leur décision comme ils l'ont fait, c'est qu'on leur a donné à entendre qu'il s'agissait non de creanda potestate leges ferendi circa matrimonium et divor* tium, sed de declarando ubinam in futuro existere debeat potestas qua jam existit in singulis par lamentis provincialibus auctoritate gubernii anglici. Or, voilà ce que je conteste. Je sais bien qu'en Angleterre, la Chambre des Lords est investie de deux pouvoirs, l'un législatif et l'autre judiciaire; et que, en vertu de son pouvoir judiciaire, elle a souvent prononcé des divorces. Mais ici, au Canada, notre législature n'a pas ce pouvoir judiciaire, quoiqu'elle l'ait usurpé dans une couple de cas. Il s'agit donc de créer ce pouvoir et non simplement de le transférer. s AAM, Lettres de $M^{\text {gr }}$ Bourget, 14, 324-325.

9 AAM, Lettres de $M^{\text {or }}$ Bourget, 16, 103. Lettre publiée dans La Minerve du 15 juillet 1867. 
respecter, auquel ils doivent obéir. Ainsi le veut la doctrine de l'Eglise, dont $\mathrm{M}^{\mathrm{gr}}$ Bourget est le gardien à Montréal et dont il doit assurer le triomphe. Tâche délicate et difficile, car Montréal avait été le centre de résistance : trois journaux s'étaient déclarés les adversaires irréductibles du projet : Le Pays, L'Ordre et le True Witness. Si, après la sanction impériale, Le Pays, organe de l'Institut Canadien, continue son opposition, L'Ordre et le True Witness se rallient à la Confédération. Et il faut voir là, nous n'en doutons pas, l'influence personnelle de $\mathrm{M}^{\mathrm{gr}}$ Bourget sur la direction de ces journaux. La Confédération n'est pas parfaite, écrit en substance George Clerk dans le True Witness; mais puisque les évêques n'y trouvent pas à redire, nous pouvons bien penser de même. Il serait injuste de la condamner avant de l'avoir vue à l'œuvre. Et faisons de notre mieux pour qu'elle fonctionne bien ${ }^{10}$. L'Ordre avait soutenu sur tous les tons que la Confédération entraînerait la ruine et la mort du Canada français. Il écrit aujourd'hui :

Nous avons toujours cru que la race française au Canada pouvait se suffire à elle-même; voilà pourquoi nous avons la ferme conviction que, même sous la Confédération, elle ne périra pas; l'union étroite des véritables patriotes sera pour elle une garantie 11 .

Le True Witness et L'Ordre entretenaient d'excellentes relations avec l'évêque de Montréal; ils l'ont certainement consulté sur la ligne de conduite à tenir. Ils ont accepté sa direction, et, sous leurs propres responsabilités, c'est la pensée de $\mathrm{M}^{\mathrm{gr}}$ Bourget qu'ils communiquent à leurs lecteurs.

Que la Confédération fût pour $\mathrm{M}^{\mathrm{gr}}$ Bourget un problème d'ordre exclusivement politique, nous en avons la preuve dans la circulaire que, le 25 mai, il adresse à son clergé sur la conduite à tenir pendant les prochaines élections:

Nous devons avoir dans un temps plus ou moins éloigné de nouvelles élections. Notre conduite sera alors ce qu'elle a été invariablement, c'est-à-dire que nous demeurerons en dehors de toute lutte électorale et de tout esprit de parti.

C'est le règlement disciplinaire du second concile provincial de Québec, en 1854, qui nous trace cette ligne de conduite par ces paroles: - Le clergé doit, dans sa vie publique et privée, demeurer neutre - dans les questions qui ne touchent en rien aux principes religieux. . Telle a toujours été d'ailleurs la règle suivie par nos Pères; et nous ne pouvons trouver un meilleur exemple.

Lorsque les principes religieux sont exposés, les évêques ne manquent pas d'élever la voix, avec le respect dû aux autorités, comme vous avez pu l'observer plusieurs fois. Ce serait donc à eux à donner l'éveil, s'il fallait encore en venir là. En attendant, chacun reste à son poste, dans une parfaite neutralité 12 .

Dans cette circulaire, on ne trouve pas davantage le mot Confédération. Mais c'est bien à elle que pense l'évêque quand, se basant toujours sur les Pères du concile de 1854, il écrit :

10 Le Courrier du Canada, 20 mars 1867.

11 Le Courrier du Canada, 8 avril 1867.

12 Mand. des év. de Montréal, V, 212-214. 
Un de ces vrais principes [que le clergé doit inculquer au peuple] pour tous les catholiques sincères est que tous les sujets sont obligés en conscience de se soumettre à tout gouvernement légitimement établi; et que ce serait un excès condamnable de travailler à le renverser par la violence ou autres mauvais moyens ${ }^{13}$.

Et c'est une allusion discrète aux événements de 1837 qu'il faut voir dans le paragraphe suivant :

Telles sont les règles pratiques qu'il nous faut suivre nous-mêmes et faire observer aux fidèles confiés à nos soins. Appliquons-nous donc à bien instruire le peuple des devoirs qu'il aura à remplir dans le temps des élections, avant que les esprits ne soient échauffés. Car, l'expérience du passé doit nous avertir de ce qui arrivera alors, si cette sage précaution n'est pas prise longtemps d'avance ${ }^{14}$.

C'est le premier document public inspiré par la Confédération à un évêque du Bas-Canada. Les autres évêques ne tarderont pas à parler. Mais entre l'attitude de $\mathbf{M}^{\text {gr }}$ Bourget et celle de ses confrères, il y a une double différence. La circulaire de l'évêque de Montréal ne s'adresse qu'au clergé, non aux fidèles. Les documents des autres évêques s'adressent directement aux fidèles, ils leur seront communiqués du haut de la chaire. $\mathbf{M}^{\mathrm{gr}}$ Bourget signifie à son clergé que la Confédération est le gouvernement légitime, auquel tous sont tenus de se soumettre. Ses confrères ne se contentent pas de cette sèche affirmation. Ils font l'éloge de la Confédération, ils affirment qu'elle ne présente aucun danger du point de vue religieux, qu'elle est l'aurore d'une ère de prospérité et de grandeur pour le Bas-Canada ${ }^{15}$.

$\mathrm{M}^{\mathrm{gr}}$ Bourget en restera-t-il là ? Osera-t-il lui aussi parler directement au peuple? Il a longtemps hésité. Le $1^{\text {er }}$ juin, il avait publié un Mandement concernant le dix-huitième anniversaire séculaire du martyre de saint Pierre et de saint Paul, apôtres. Le diocèse de Montréal ne pouvait rester étranger à un événement si glorieux pour l'Erlise. Il sera représenté à Rome par M. Truteau, vicaire-général. C'est encore trop peu. On imitera à Montréal ce qui se fera à Rome; précédée

13 Ibid.

14 Ibid.

15 La Minerve se fait un devoir de reproduire les mandements ou pastorales des évêques du Bas-Canada sur le sujet. Et elle y ajoute, il va sans dire, un petit couplet clérical. - Les évêques des Provinces Maritimes n'avaient pas attendu la sanction royale pour se prononcer. La Minerve du 27 janvier 1865 publie en traduction la lettre adressée par $\mathbf{M}^{\mathrm{gr}}$ Connolly, archevêque d'Halifax au Morning Chronicle de cette ville. En voici le dernier paragraphe : Après la plus mûre considération du sujet et tous les arguments que j'ai entendus de tous côtés, dans le cours du dernier mois, c'est ma conviction la plus profonde que la confédération est nécessaire, qu'elle est la mesure seule qui, avec le secours de la Providence, peut assurer l'ordre social, la paix, la liberté rationnelle et tous les bienfaits dont nous jouissons maintenant, sous le gouvernement le plus doux et les institutions du pays le plus libre et le plus heureux du monde." La Minerve du 28 février 1865 donne des extraits des lettres des évêques des quatre provinces maritimes, tous favorables à la confédération. Le 22 mars de la même année, elle publie la lettre adressée par $\mathrm{M}^{\mathrm{gr}}$ Mulloch, évêque de Terre-Neuve au Times de Saint-Jean. Enfin, le 27 avril 1866, elle offre à ses lecteurs une traduction de la lettre de $\mathbf{M}^{\mathrm{gr}} \mathbf{M c K i n}$ non, évêque d'Arichat. 
par une neuvaine dans toutes les paroisses du diocèse, la fête des saints Apôtres sera célébrée avec un éclat inaccoutumé; et l'évêque interrompra sa visite pastorale pour venir donner au peuple la bénédiction pontificale ${ }^{16}$. Tel était le programme; mais de Saint-Isidore, $M^{\mathrm{gr}}$ Bourget écrit à son secrétaire, le 20 juin :

Après avoir lu dans $L a$ Minerve du 17 courant, l'article intitulé Fête de la Confédération, je me détermine à ne plus conseiller l'illumination de la St-Pierre, et à borner les honneurs à rendre à nos saints Apôtres aux offices qui se feront dans l'intérieur des églises. Je vous charge, en conséquence, d'en donner avis aux Institutions qui ont été invitées à le faire, en leur disant, sans autre préambule, qu'il ne se fera rien de nouveau, le jour de la St-Pierre, pour célébrer le $18^{\circ}$ anniversaire séculaire de leur [sic] martyre, si ce n'est un redoublement de piété au fond des âmes et dans l'intérieur des églises...

Nous allons commencer tout à l'heure notre neuvaine. J'espère que les bonnes prières qui vont se faire dans tout le diocèse feront plus de bien à la Confédération que le plus beau mandement. Du moins, personne ne pourra s'en fâcher ${ }^{17}$.

Le comité des citoyens ayant décidé de célébrer avec éclat le $1^{\circ r}$ juillet, date d'entrée en vigueur de la Confédération, $M^{\mathrm{gr}}$ Bourget ne veut pas que les solennités religieuses extérieures du 29 juin entrent en conflit ou en comparaison avec les manifestations profanes du $1^{\text {er }}$ juillet. Parce qu'elle est le gouvernement légitime, la Confédération a droit aux prières qui se font à l'intérieur des églises; parce que, dans sa pensée, son avènement est un fait d'ordre exclusivement politique, il n'y a pas lieu d'en faire le sujet d'un Mandement ou d'une Pastorale. - Du moins, a-t-il écrit, personne ne pourra s'en fâcher." Mais ce n'est pas là la raison profonde de son abstention. L'on sait assez que l'évêque de Montréal ne craignait pas de "fâcher " ses fidèles, quand il s'agissait de revendiquer les droits de la foi ou de la morale. Seulement il ne s'agit pas de cela ici; car ni l'une ni l'autre ne sont menacées.

L'heure allait pourtant venir où la morale imposerait à $\mathrm{M}^{\mathrm{gr}}$ Bourget de parler; la morale menacée non pas par la Confédération, mais par les élections qui marquent son entrée en vigueur. Lettre Pastorale indiquant des prières publiques à l'occasion des prochaines élections ${ }^{18}$, tel est le titre de cette instruction aux fidèles. Et les premiers mots en indiquent clairement le sens et le but : " Partout, N.T.C.F., l'on n'entend parler que d'élections; c'est le sujet ordinaire de presque toutes les conversations. On s'en entretient dans les villes et les campagnes, dans les assemblées publiques et les réunions particulières, dans les maisons et les rues, sur les chemins de fer et les bateaux à vapeur... Les esprits en sont tout préoccupés; et il est fort à craindre qu'ils ne finissent par se diviser et en être ulcérés.

La Pastorale est tout simplement l'adaptation aux fidèles de la circulaire au clergé du 25 mai. L'évêque ne craint pas d'affirmer, à la

16 Mand. des év. de Montréal, V, 215-228.

17 AAM, Voyage à Rome, 1869-1870, V, cart. 15.

18 Mand. des év. de Montréal, V, 236-244. 
face de son diocèse, son indépendance à l'égard des partis politiques. Il se maintient dans la région sereine des principes et s'abstient de toute allusion aux personnes et partis en présence :

Car, remarquez-le bien, il y a une grande différence entre cette direction : Votez ou ne votez pas pour tel candidat, et cette autre : Votez pour celui qui, dans votre âme et conscience, vous paraît qualifié pour soutenir les intérêts de la Religion et de la Patrie ${ }^{19}$.

Parmi les vrais principes qui doivent guider les électeurs dans le choix de leurs représentants, il en était un qu'il était particulièrement opportun de rappeler en 1867 : c'était l'attitude de l'Eglise envers les gouvernements légitimes. Car aux yeux de $\mathrm{M}^{\mathrm{gr}}$ Bourget, la Confédération est désormais le gouvernement légitime; et il ne manque pas de candidats qui continuent de s'y opposer, qui en demandent le rejet. Que dira donc l'évêque de Montréal ?

$\mathrm{Si}$ vous y faites attention, vos pasteurs ne font pas autre chose en cela que vous rappeler cette sentence sortie de la bouche de la Sagesse éternelle : Rendez donc à César ce qui est à César et à Dieu ce qui est à Dieu. Car Dieu a établi dans le monde la société religieuse et la société civile pour que d'un commun accord elles travaillent au bonheur des peuples. Il s'ensuit que ces deux sociétés sont tenues de se soutenir réciproquement sans aucunement anticiper sur les droits de l'une ou de l'autre.

Cet ordre, donné à tous les enfants de l'Eglise par son divin Fondateur, règle tous les devoirs qu'ils ont à remplir envers le gouvernement, quelles que soient sa nature et sa forme, dès qu'il est légitimement constitué. Aussi, cette Eglise a-t-elle toujours accepté et accepte-t-elle encore les divers gouvernements qui se succèdent les uns aux autres. Ainsi, sans en aller chercher d'exemple ailleurs, l'Eglise du Canada est demeurée soumise au Roi de France jusqu'à ce que la divine Providence fît passer ce pays sous la domination britannique. Elle accepta alors, sans aucune contrainte et par devoir de conscience ce nouveau gouvernement sous lequel elle a heureusement joui de tous ses droits religieux. Vint ensuite la Constitution, puis l'Union des deux Provinces. L'Eglise s'y soumit et prêcha à ses enfants l'obéissance à l'autorité constituée. Aujourd'hui, elle accepte sans réplique le Gouvernement fédéral, parce qu'il émane de la même Autorité. Ainsi, vous le voyez, N.T.C.F., le changement de gouvernement n'a pas été pour nos pères, et ne peut non plus être pour nous une raison de résister au pouvoir existant. Notre-Seigneur nous a fait un devoir, quand il a tracé à son Eglise la conduite qu'elle aurait à tenir dans tous les siècles, envers toute espèce de gouvernement, par ces paroles mémorables: Rendez à César ce qui est à César. Car, il est bien à remarquer que César avait changé le gouvernement de l'ancienne Rome, qui était républicain, pour y sybstituer l'Empire Romain, auquel appartenait alors la Judée par droit de conquête. Quelques-uns parmi les Juifs n'admettaient pas ce gouvernement comme légitime, et c'était se rendre impopulaire que de se déclarer pour le gouvernement existant. Cependant, le divin Législateur, sans plus d'explications, veut qu'on l'accepte et qu'on lui obéisse 20 .

Après avoir rappelé la doctrine de saint Paul sur le même sujet, l'évêque adresse à tout le diocèse la directive qu'il avait donnée, deux mois plus tôt, au seul clergé : «Un de ces vrais principes pour tous

19 Ibid.

20 Ibid. 
les catholiques sincères est donc, N.T.C.F., que tous les sujets sont obligés en conscience de se soumettre à tout gouvernement légitimement établi; et que ce serait un excès condamnable de travailler à le renverser par la violence ou autres mauvais moyens ${ }^{21}$."

Tout cela est dit d'un ton à la fois serein et ferme. Aucun jugement n'est porté sur les avantages ou les désavantages, les mérites ou les démérites de la Confédération considérée comme telle. Pour l'évêque de Montréal le problème n'est pas là; il est dans l'attitude et dans l'enseignement de l'Eglise et de son divin Chef à l'égard des pouvoirs légitimes. Les catholiques sincères, ceux qui sont plus attachés à l'Eglise qu'à un parti politique ou à une forme de gouvernement, ne peuvent se méprendre sur la ligne de conduite à tenir : "Aujourd'hui, elle [l'Eglise] accepte sans réplique le Gouvernement fédéral. »

Les directives de $\mathrm{M}^{\mathrm{gr}}$ Bourget ne diffèrent donc pas de celles des autres évêques du Bas-Canada. Sa façon d'aborder le problème est autre, parce qu'à Montréal l'état des esprits est autre. Ici, depuis trois ans, au vu et au su de l'évêque, deux journaux catholiques ont fait campagne contre la Confédération; ici plus qu'ailleurs les adversaires du projet se recrutent parmi les catholiques et non parmi les moindres. Ce n'est pas en heurtant directement ces hommes de bonne volonté aveuglés par l'esprit de parti, que l'évêque accomplira son devoir de Pasteur des âmes. C'est en éclairant les esprits, en rappelant la doctrine de l'Eglise sur les gouvernements légitimes, quelles qu'en soient la nature et la forme. Quand Jésus a dit : Rendez à César ce qui est à César, il n'a pas approuvé toute l'administration de César, il n'a pas exalté sa personne. Il a constaté un fait et il en a tiré les conséquences. Ajoutons que si la manière de $\mathrm{M}^{\mathrm{gr}}$ Bourget lui est inspirée par les circonstances particulières où il se trouve, elle reste conforme à l'attitude de neutralité envers les partis politiques, qu'il avait adoptée dès le début.

Cette prudence de $\mathrm{M}^{\mathrm{gr}}$ Bourget a paru regrettable à certains de ses confrères. Le 12 octobre $1867, \mathrm{M}^{\mathrm{gr}}$ Charles Larocque, évêque de Saint-Hyacinthe, signale à $\mathrm{M}^{\mathrm{gr}}$ Bourget le parti que les libéraux avancés, dont la mauvaise foi et la malice sont évidentes, tirent de sa Pastorale. Ils continuent de répéter qu'il y a division chez les évêques au sujet de la Confédération, que $M^{\mathrm{gr}}$ Bourget la réprouve, qu'il aurait été heureux du triomphe des anti-fédéralistes aux récentes élections; ils interprètent son silence comme une condamnation des autres évêques. Enfin, en son nom, au nom de quelques évêques et de plusieurs prêtres bien pensants et distingués, $M^{\mathrm{gr}}$ Larocque exprime le désir que l'évêque de Montréal fasse, pour mettre fin au scandale, une déclaration publique plus explicite ${ }^{22}$.

$\mathrm{M}^{\mathrm{gr}}$ Bourget lui écrit trois jours plus tard :

En réponse à la vôtre du 12 courant, je crois devoir déclarer, dans la sincérité de mon âme, que je pense avoir dit, dans ma circulaire et dans ma Lettre Pastorale, sur lesquelles Votre Grandeur a

21 Ibid.

22 AAM, Diocèse de Saint-Hyacinthe. 
attiré mon attention, tout ce que je pouvais et devais dire sur la Confédération pour la direction du clergé et l'instruction du peuple. Ces lettres ont paru produire leur effet; et je crois que tous ont compris que c'était pour eux un devoir de conscience de se soumettre à la Confédération et qu'il leur fallait voter en conséquence. A la vérité, ils n'ont pas tous été fidèles à cet enseignement, et je suis le premier à le déplorer. Au reste, $\mathbf{M}^{\mathrm{gr}}$ l'archevêque [Baillargeon] a bien voulu me témoigner, à Berthier, qu'il approuvait ma manière de procéder, lorsque je me suis permis de lui demander s'il ne serait pas à propos de donner une nouvelle directive au sujet des mauvais journaux qui, aujourd'hui s'élèvent avec fureur contre les évêques et le clergé.

Vous savez ce que j'en pense, pour ma part; et l'on aura, dans l'occasion, la preuve que je suis maintenant, comme je l'ai toujours été dans le passé, pour maintenir le gouvernement établi. C'est donc à tort que l'on cherche à faire croire que je ne suis pas pour la Confédération, sous prétexte que je me suis expliqué autrement qu'eux dans les documents précités. Un de nos chauds conservateurs, qui est membre des deux chambres, fédérale et locale, disait dans une certaine réunion que l'évêque de Montréal, dans ses lettres sur la Confédération, avait pris une position qui lui permettait de défendre ses principes sous n'importe quelle administration, pourvu qu'elle soit vraiment constitutionnelle 23 .

On ne pouvait mieux dire.

\section{ConCLUSION}

Après la sanction impériale qui faisait de la Confédération le gouvernement légitime du pays, l'attitude de $\mathrm{M}^{\mathrm{gr}}$ Bourget nous est donc clairement connue. Saura-t-on jamais ce qu'il pensait aussi longtemps que le sujet fut laissé à la libre discussion des hommes ? Une tradition s'est établie suivant laquelle, dans son for intérieur, il aurait été opposé à la Confédération. A notre connaissance, il n'existe aucun document, aucune parole émanant de lui qui permette de trancher le débat. Cette tradition ne manque pas de vraisemblance. En 1839-1840, $\mathrm{M}^{\mathrm{gr}}$ Lartigue avait organisé, tant au Canada qu'à Londres, une campagne de résistance au projet d'Union, parce qu'il y voyait un danger pour la liberté de l'Eglise et la foi traditionnelle des Canadiens français ${ }^{24}$. Cette crainte, qui paraissait justifiée, $\mathbf{M}^{\mathrm{gr}}$ Bourget l'avait lui-même manifestée au gouverneur Poulett Thomson ${ }^{25}$. En 1865, il était permis à un évêque du Bas-Canada de nourrir les mêmes inquiétudes. La présence du Haut-Canada et des Provinces Maritimes dans la Confédération n'étaitelle pas de nature à diminuer l'importance des catholiques sur la législation du pays. Quant au gouvernement local, on ne savait pas jusqu'à quel point il serait le rempart de nos institutions, de nos lois et de notre foi.

Dans le Bas-Canada, la Confédération devait surtout sa popularité au prestige de Georges-Etienne Cartier. "Nous avons foi en nos chefs * avait dit La Minerve en 1864; et le prestige de Cartier n'avait cessé

23 AAM, Lettres de $M^{\text {or }}$ Bourget, 16, 458-459.

24 RHAF, sept. 1954 (vol. VIII, $\mathrm{n}^{\circ}$ 2): "Les évêques du Bas-Canada et le projet d'Union (1840).,

25 BRH, 1956, 149-156 : - Deux entretiens de $\mathbf{M}^{\text {rr }}$ Bourget avec Poulett Thomson sur le projet d'Union. , 
de grandir. Avec un tel homme à la tête du gouvernement, le BasCanada n'avait rien à craindre ni pour sa langue ni pour sa foi.

Cependant, $\mathrm{M}^{\mathrm{gr}}$ Bourget était libre de tout enthousiasme exagéré à l'égard de Georges-Etienne Cartier. Il le tenait responsable des articles gallicans de notre législation civile. Quand, en 1866, l'évêque de Montréal entreprend d'exécuter les décrets de Rome sur le démembrement de la paroisse de Notre-Dame, qui trouve-t-il pour lui barrer la route? La Minerve, l'organe de Cartier. Dans ce procès célèbre, quel est l'avocat des Sulpiciens contre l'évêque ? Georges-Etienne Cartier. De Londres où ils sont allés soutenir de leur présence le bill de la Confédération, Cartier et ses collègues catholiques se rendent à Rome, au début de 1867. Pourquoi ? Autant, sinon plus, pour défendre les Sulpiciens contre l'évêque que pour satisfaire leur dévotion personnelle.

Que $\mathrm{M}^{\mathrm{gr}}$ Bourget ait vu d'abord dans la Confédération un danger pour le Bas-Canada catholique, la chose nous paraît vraisemblable. Le champion de celle-ci, le gallican Cartier n'est pas exempt de tout reproche; il n'en impose pas à $\mathbf{M}^{\mathrm{gr}}$ Bourget; et c'est le moins que l'on puisse dire.

Mais, conviction personnelle et sentiments humains n'ont pas de prise sur sa volonté, dès qu'ils entrent en conflit avec la doctrine de l'Eglise. A partir du jour où par décision de Londres, la Confédération devient le gouvernement légitime, $\mathrm{M}^{\mathrm{gr}}$ Bourget l'accepte sans arrièrespensées et sans faux-fuyants. Son adhésion n'est pas seulement extérieure; elle est sincère, entière, loyale.

Léon Pouliot, s.j.

Professeur d'histoire de l'Eglise, Montréal, P.Q. 